\title{
A Simplified Empirical Modeling of Electrophysiological Activity in a Bundle of Myelinated Nerve Fibers
}

\author{
A. G. Pizá, F. D. Farfán, A. L. Albarracín, F. A. Lucianna, J. H. Soletta and C. J. Felice
}

\begin{abstract}
In vivo or in vitro electrophysiological characterization is a standard procedure to know specific aspects of the sensory and motor fibers conduction. In this work we propose a simplified empirical modeling that can predict the electrical activity evocated in a nerve by different experimental conditions. This approach includes physical and chemical concepts about the generation and propagation of myelin fiber action potentials, volume conduction and medium bioelectric properties; this was implemented by using simple qualitative parameters. The validation included the qualitative analysis of compound action potentials (CAPs) obtained from frog sciatic nerve in different experimental conditions and CAPs recordings obtained from infraorbital nerve in rats. The results reveal an adequate model fit to the CAPs waveform experimentally obtained. The intuitive parameters used in our approach facilitate the implementation and results interpretation, at the same time that provides versatility and robustness.
\end{abstract}

Keywords- Compound action potential, differential recording, electrophysiological characterization

\section{INTRODUCTION}

$\mathrm{L}$ A CARACTERIZACIÓN electrofisiológica in-vitro, o in- vivo, de fibras mielínicas periféricas es un procedimiento estándar que permite determinar aspectos funcionales del nervio, tales como, velocidad de conducción, distribución de fibras mielínicas/no-mielínicas, y características refractarias temporales de las fibras nerviosas [1]. Para ello, la morfología temporal del potencial de acción compuesto (CAP, por sus siglas en inglés), registrado en un haz de fibras nerviosas, es analizada [2]. La modelización matemática del CAP ha permitido revelar aspectos funcionales específicos, los cuales muchas veces resultan experimentalmente dificultosos de acceder, tales como la contribución electrofisiológica de diferentes haces de fibras mielínicas [3].

El CAP es comúnmente modelado como una superposición lineal de potenciales de acción de fibra única (SFAPs, por sus siglas en inglés). Algunas aproximaciones, frecuentemente referidos como modelos físicos, determinan analíticamente el

A. G. Pizá, Universidad Nacional de Tucumán (UNT), San Miguel de Tucumán, Tucumán, Argentina, piza.ag@gmail.com

F. D. Farfán, Universidad Nacional de Tucumán (UNT), San Miguel de Tucumán, Tucumán, Argentina, ffarfan@herrera.unt.edu.ar

A. L. Albarracín, Universidad Nacional de Tucumán (UNT), San Miguel de Tucumán, Tucumán, Argentina, anaalbarracin@gmail.com

F. A. Lucianna, Universidad Nacional de Tucumán (UNT), San Miguel de Tucumán, Tucumán, Argentina, facundolucianna@gmail.com

J. H. Soletta, Universidad Nacional de Tucumán (UNT), San Miguel de Tucumán, Tucumán, Argentina, jorge.soletta@gmail.com

C. J. Felice, Universidad Nacional de Tucumán (UNT), San Miguel de Tucumán, Tucumán, Argentina, cfelice@gmail.com
SFAP usando la teoría de conducción por volumen y descripciones de la actividad eléctrica intracelular [4], [5], mientras que otras, referidos como modelos empíricos, establecen la forma de onda del SFAP y se asumen ciertas condiciones de propagación sobre el haz de fibras [6]-[9]. En todos los casos, las formulaciones requeridas tienen implementaciones matemáticamente complejas las cuales dificultan una adecuada parametrización del modelo de CAP.

En este trabajo proponemos un modelo simplificado de la actividad electrofisiológica de un haz de fibras mielínicas, el cual permite predecir la forma de onda del CAP registrado en diferentes condiciones experimentales. Nuestra aproximación empírica permite simular condiciones experimentales tales como: adquisición del CAP en forma monopolar / diferencial, variación de forma de onda del CAP en función de la posición y distancia entre-electrodos de registro, cambios del CAP en función de la morfología intrínseca del impulso nervioso, contribución electrofisiológica de fibras anatómicamente diferentes y el efecto de los cambios de conductividad eléctrica del intersticio. El modelo reúne conocimientos físicoquímicos de la generación y propagación de potenciales en fibras mielínicas, conducción por volumen, propiedades eléctricas del medio, y son implementados a través de simples parámetros cualitativos.

La validación fue realizada a través del análisis cualitativo de simulaciones de CAPs obtenidos en el nervio ciático de sapo bajo diferentes condiciones experimentales, y a través de registros experimentales de CAPs registrados en el nervio infraorbitario de rata. Los resultados revelan un adecuado ajuste del modelo a registros CAP obtenidos experimentalmente. La intuitiva parametrización, utilizada por nuestra aproximación, facilita su implementación e interpretación de resultados, del mismo modo que le proporciona gran versatilidad y robustez.

\section{MATERIALES Y MÉTODOS}

Un impulso nervioso, Vm, es capaz de propagarse a lo largo de un axón mielínico a una velocidad de conducción, $v$, la cual es directamente proporcional al diámetro de la fibra [1]. Tal propagación es realizada de un modo saltatorio, es decir, que el impulso nervioso se propaga desde un nodo a otro sin sufrir alteraciones de amplitud. Así, Vm podría ser observado en cada uno de los nodos de Ranvier, pero en diferentes instantes de tiempo (Fig. 1A). Tal propagación es realizada en un modo saltatorio, es decir, que el impulso nervioso se propaga desde un nodo a otro sin sufrir alteraciones de amplitud [10]. Si un electrodo extracelular, $e$, es colocado en las cercanías de una 
fibra, podría observarse el SFAP, el cual, matemáticamente, puede ser calculado como sigue:

$$
\begin{gathered}
\text { SFAP }=\sum_{i=\infty}^{\infty} V_{m}\left(t-\frac{x_{i}}{v}\right) \cdot W_{i} \\
W_{i}=\left\{\begin{array}{ccc}
W_{\max }\left(\frac{x_{i}-d_{e}}{d}\right)+W_{\max } & \text { if } & d_{e}-d \leq x_{i}<d_{e} \\
-W_{\max }\left(\frac{x_{i}-d_{e}}{d}\right)+W_{\max } & \text { if } & d_{e} \leq x_{i} \leq d_{e}+d \\
0 & \text { othercase }
\end{array}\right.
\end{gathered}
$$

Donde $W_{i}$ es un factor de ponderación que depende de la conductividad longitudinal y el área transversal del intersticio, como así también de la distancia entre el i-ésimo nodo y el electrodo extracelular [6]. En la ec. 2, $x_{i}$ es la distancia desde un hipotético origen en $\mathrm{x}=0$ hasta el $i$-ésimo nodo, $d_{e}$ es la distancia computada desde $\mathrm{x}=0$ hasta el electrodo extracelular, $d$ es la distancia entre la mínima y máxima atenuación de $V_{m}$ (Fig. 1B). $W_{\max }$ proporciona el mínimo factor de atenuación y ocurre en la posición longitudinal, cuya distancia entre el $i$-ésimo nodo y el electrodo de registro es mínima (posición $x_{i}$ en la Fig. 1B).

En el modelo propuesto, se consideran $\mathrm{N}$ fibras mielínicas, por lo que $W_{\max }$ es una función de la distancia perpendicular entre la j-ésima fibra y el electrodo de registro $\left(d f_{j}\right.$ en la Fig. $1 \mathrm{C})$.

$$
W_{\max }(d f)=\left\{\begin{array}{ccc}
1-\frac{d f}{\Theta_{b}} \alpha & \text { for } \quad 0 \leq d f<\frac{\Theta_{b}}{\alpha} \\
0 & \text { othercase }
\end{array}\right.
$$

Donde $\Theta_{b}$ es el diámetro del haz de fibras nerviosas, y $\alpha$ es una constante de proporcionalidad la cual está relacionada a la conductividad transversal del intersticio. La ec. 3 es una recta de pendiente negativa y ordenada al origen 1 , es decir que $W_{\max }=1$ para $d f=0$ (esto corresponde a una fibra que está en contacto con el electrodo de registro). Por otro lado $W_{\max }=0$, para una $d f=\Theta_{b} / \alpha$ (correspondiente a la actividad de la fibra más lejana). Luego para $\mathrm{N}$ fibras, el CAP es computado como sigue:

$$
\begin{gathered}
C A P_{e 1}=\sum_{j=1}^{N} S F A P_{j} \\
C A P=C A P_{e 1}-C A P_{e 2}
\end{gathered}
$$

Donde SFAP es el potencial generado por la $j$-th fibra $\mathrm{y}$ $C A P_{e l}$ es el potencial de acción compuesto registrado por el electrodo el (Fig. 1C). Finalmente, el CAP diferencial es obtenido con la ec. 5 .

\section{A. Simulaciones}

El modelo propuesto fue sometido a simulaciones específicas con el fin de evaluar su comportamiento en diferentes condiciones experimentales. Dos haces de fibras

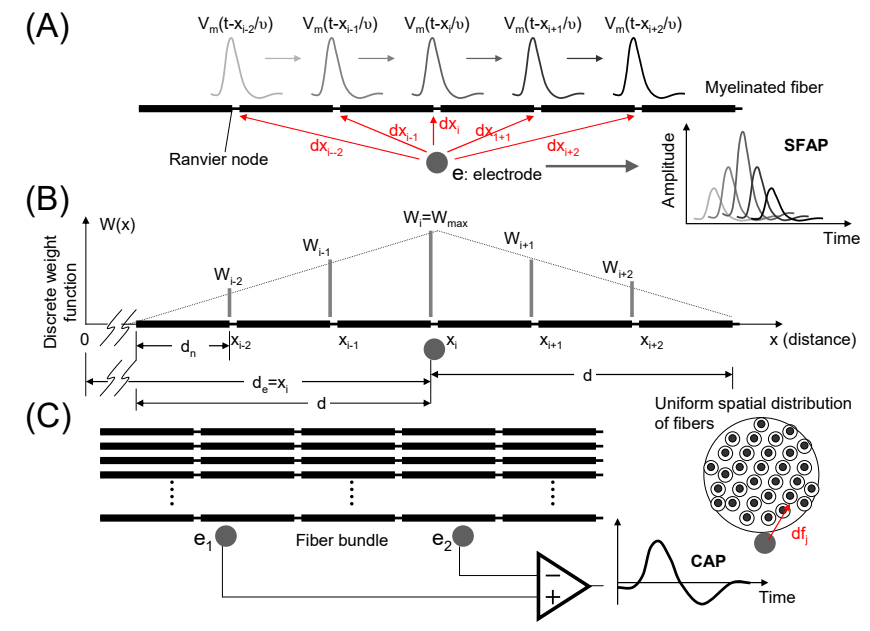

Figura 1. Modelo simplificado de la actividad aferente multifibra. (A) Modelización del SFAP registrado a través de un electrodo de registro, e. La atenuación del impulso nervioso $\mathrm{Vm}$, depende de la distancia entre el i-th nodo y el electrodo de registro. (B) Factor de atenuación en función de la distancia al electrodo de registro. El origen, $\mathrm{x}=0$, es considerado como el punto de partida, en el cual se desencadena el impulso nervioso, Vm. (C) Consideraciones esquemáticas para la modelización de un CAP en un haz de fibras mielínicas. La disposición espacial de fibras tiene una distribución uniforme en toda el área transversal del haz.

mielínicas fueron considerados: 1) el nervio ciático de sapo, y 2) el nervio infraorbitario de rata. La implementación del modelo, en ambos casos, tuvo en cuenta las siguientes consideraciones, según Reutskiy et al. y Wang et al. [11], [12]: el axón tiene simetría cilíndrica, la sección transversal de la fibra es constante, los nodos de Ranvier están igualmente espaciados, las corrientes iónicas activas están concentradas en el nodo de Ranvier, la membrana internodal es totalmente pasiva, la sección transversal del axón es isopotencial, las concentraciones de iones son constantes, las fibras son paralelas y los nodos de Ranvier están alineados (Fig. 1C), las fibras no son idénticas, no hay acoplamiento radial directa entre fibras vecinas.

Simulación de un nervio ciático. Los parámetros morfológicos del nervio ciático fueron obtenidos de Wijesinghe et al. [13]. Así, el nervio simulado tiene un diámetro de $1500 \mu \mathrm{m}, 4000$ fibras mielínicas y distancia entre nodos $2000 \mu \mathrm{m}$. Los diámetros de las fibras mielínicas tienen una distribución de frecuencias la cual es directamente proporcional a la velocidad de conducción (Fig. 2A). Los electrodos de registros $e_{1}$ y $e_{2}$ son colocados a distancias $d_{e l} \mathrm{y}$ $d_{e 2}$, respectivamente, de manera que la distancia mínima entre $e_{1}$ y la j-ésima fibra es cero (ídem para $e_{2}-$ Fig. 2B). En estas condiciones, la simulación determina los CAPs observados por ambos electrodos (modos monopolar y diferencial), originados por la aplicación de un estímulo supramaximal en $\mathrm{x}$ $=0$, el cual despolariza la totalidad de las fibras, desencadenando así la propagación de $V_{m}$ a lo largo de cada una de ellas. Diferentes situaciones experimentales fueron simuladas: 
a) Variación de la distancia inter-electrodo $\left(\left|d_{e 1}-d_{e 2}\right|\right) \cdot e_{1}$ fue colocado a $d_{e l}=1 \mathrm{~cm}$, y $e_{2}$ fue colocado a distancias de modo que $\left|d_{e 1}-d_{e 2}\right|$ fue 1, 2, 3, 4, 5 y $6 \mathrm{~cm}$ (Fig. 2B).

b) Variación de la posición longitudinal de los electrodos de registro. La distancia inter-electrodo se mantuvo constante $\left(\left|d_{e 1}-d_{e 2}\right|=1 \mathrm{~cm}\right)$, mientras que el par de electrodos fue desplazado longitudinalmente a 1, 2, 3, 4, 5 y $6 \mathrm{~cm}$ (Fig. 2C).

c) Variación de apertura longitudinal de $W_{j}$. Se varió la distancia entre el mínimo y máximo valor de la función de ponderación, $W_{j}$, $(d$ en la Fig. 1B). $d$ fue preestablecido de acuerdo a la cantidad de nodos abarcados por $W_{j}$, siendo ésta la función de ponderación perteneciente a la fibra cuya $d f_{j}=0$ (Fig. 1C). La cantidad de nodos considerados fueron, 10,6 y 2 nodos. De esta manera, para una $d_{n}=2000 \mu \mathrm{m}, d$ fue igual a 2, 1.2 y $0.4 \mathrm{~mm}$, respectivamente (Fig. 2D).

d) Variación de la duración temporal de $V_{m}$. Las duraciones evaluadas fueron $0.3,0.4,0.6$ y $1.2 \mathrm{~ms}$ (Fig. 2E).

e) Variación de la conductividad transversal del intersticio. La conductividad del intersticio fue cualitativamente evaluada variando la función $W_{\max }$ (eq. 3) de modo que $W_{\max }(d f=0)$ fue igual a $1,3 / 4,1 / 2$ y $1 / 4$ (Fig. 2F).

Simulación de un nervio infraorbitario. Los parámetros morfológicos del nervio infraorbitario fueron obtenidos de Jacquin et al. [14]. La actividad electrofisiológica simulada fue obtenida desde un haz de $1265 \mu \mathrm{m}$ de diámetro, 5207 fibras mielínicas con distribución de frecuencia aproximadamente gaussiana de media igual a $4.5 \mu \mathrm{m}$ y

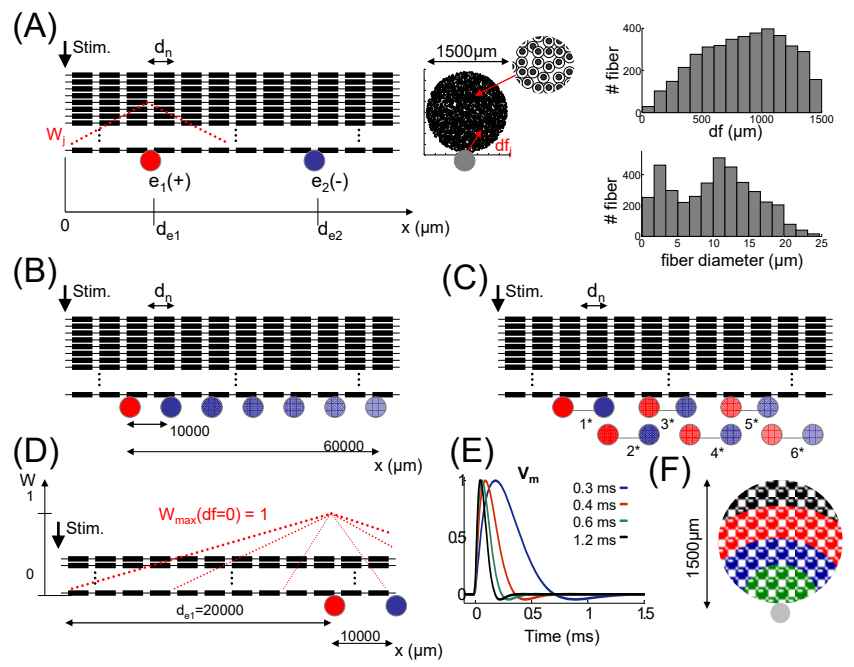

Figura 2. Situaciones experimentales simuladas. (A) Esquema de disposición general de los electrodos e1, e2, respecto a la geometría del haz. Los impulsos nerviosos, se generan en la posición $\mathrm{x}=0$ (por ej. debido a una estimulación supramaximal), y se propagan a lo largo de las fibras a velocidades de conducción específicas. Las fibras son distribuidas uniformemente en toda el área transversal del haz. El histograma superior muestra la distribución de distancias entre cada fibra y el electrodo de registro. Los diámetros de las fibras son asignados aleatoriamente y tienen una distribución específica (histograma inferior). (B) Variación de la distancia inter-electrodo. (C) Variación de la posición longitudinal de los electrodos de registro. (D) Variación de la apertura longitudinal de Wj. En este esquema solo se muestra el factor de ponderación perteneciente a la fibra en contacto con el electrodo e1 $(+)(\mathrm{dfj}=0)$. (E) Variación de la duración temporal de Vm. (F) Variación de la conductividad transversal del intersticio (variación de $\alpha$ ). desviación estándar $1.5 \mu \mathrm{m}$ (Fig. 3B). Debido a que se desconocen las distancias entre nodos, aquí se han considerado $d_{n}=500,1000,1500$ y $2000 \mu \mathrm{m}$. Las fibras fueron consideradas como distribuidas uniformemente en un área circular de $1256000 \mu \mathrm{m}^{2}$. La velocidad de conducción fue determinada experimentalmente $(34.3 \mathrm{~m} / \mathrm{s})$ a través del protocolo descrito en sección subsiguiente (validación experimental). La velocidad de conducción fue asociada a las fibras de diámetro promedio $(4.5 \mu \mathrm{m})$, estableciéndose un factor de proporcionalidad igual a $7.6(\mathrm{~m} / \mathrm{s} / \mu \mathrm{m})[1]$. Los electrodos de registros $e_{1}$ y $e_{2}$ fueron colocados a distancias $d_{e l}$ $=1.2 \mathrm{~cm} \mathrm{y} d_{e 2}=1.6 \mathrm{~cm}\left(\left|d_{e 1}-d_{e 2}\right|=4 \mathrm{~mm}\right)$.

\section{B. Validación experimental}

Para validar experimentalmente las simulaciones sobre el nervio infraorbitario se realizaron experimentos agudos en ratas Wistar macho de 300 - 350 gr, bajo anestesia general con Uretano $(150 \mathrm{mg} / \mathrm{Kg})$. Se expuso el nervio infraorbitario (IO) del lado derecho y se seccionó distalmente el nervio a la altura en que el IO ingresa a la región de las vibrisas (Fig. $3 \mathrm{~A})$.

Se insertaron, con una aguja hipodérmica, dos electrodos de estimulación (alambres de nichrome, $0.2 \mathrm{~mm}$ de diámetro; se retiró la capa aislante en alrededor de $0.5 \mathrm{~mm}$ del extremo) en el tronco del nervio infraorbitario. La distancia interelectrodos de estimulación fue de $2.5 \mathrm{~mm}$ (Fig. 3A).

Se tomaron registros de la actividad del nervio IO con electrodos bipolares de plata, colocados sobre el nervio, y separados entre sí por aproximadamente $4 \mathrm{~mm}$. La distancia entre los electrodos de estimulación y los de registros fue de $1.2 \mathrm{~cm}$. Se estimuló con pulsos rectangulares de $1 \mathrm{~Hz}(30 \mu \mathrm{s}$ de duración, supramaximales). Se tomaron 40 registros de 7 mseg cada uno y, posteriormente, se promediaron los CAPs generados.

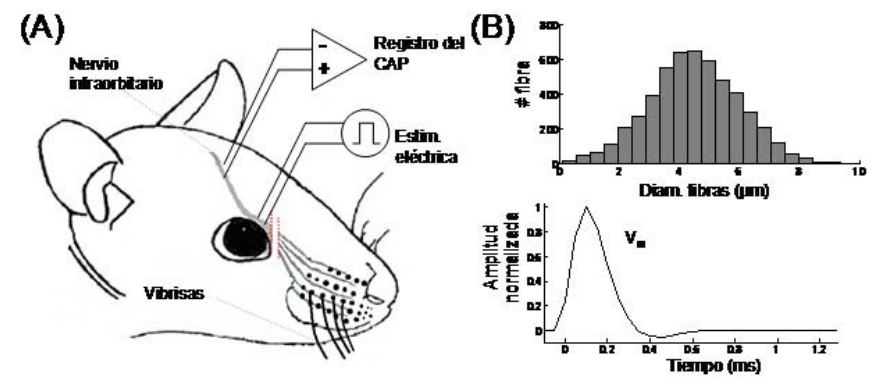

Figura 3. Validación experimental. (A) Diagrama esquemático del protocolo experimental usado para registrar los CAPs en el nervio infraorbitario. (B) Consideraciones teóricas del modelo: Arriba, histograma de la distribución de diámetros pertenecientes un haz de 5207 fibras mielínicas, abajo, impulso nervioso que se propaga a lo largo de todas las fibras del nervio infraorbitario.

\section{RESULTADOS}

El modelo propuesto en este trabajo permite estimar cualitativa y cuantitativamente la morfología de los CAPs evocados en un haz de fibras mielínicas. Para ello el modelo requiere un conocimiento previo de las características morfológicas del haz, tales como diámetro total, distribución estadística de los diámetros de todas las fibras, velocidad de 
conducción, y el impulso nervioso medido en los nodos de Ranvier.

Así mismo, son requeridos características específicas del setup experimental que se desea simular, tales como la posición de los electrodos de registro, respecto del lugar donde se genera el impulso nervioso (p.e. posición del electrodo de estimulación). En la Fig. 4A se observa el resultado de una simulación la cual reproduce la siguiente situación experimental: los electrodos de registro son colocados longitudinalmente a distancias de 10000 y $20000 \mu \mathrm{m}$ del electrodo de estimulación sobre un nervio ciático. A través de una estimulación supramaximal se desencadena la propagación de impulsos nerviosos por cada una de las fibras, los cuales se propagan a diferentes velocidades de conducción. Así, es posible determinar los CAPs monofásicos obtenidos por cada uno de los electrodos $\left(\mathrm{CAP}_{\mathrm{e} 1}\right.$ y $\left.\mathrm{CAP}_{\mathrm{e} 2}\right)$ así como el CAP diferencial. Esta simulación fue realizada considerando un impulso nervioso de $0.5 \mathrm{~ms}$ de duración y amplitud unitaria (línea gris).

La Fig. 4B muestra la simulación de CAPs diferenciales para distancias inter-electrodos incrementales. El electrodo $\mathrm{e}_{1}$ se mantiene en la posición $10000 \mu \mathrm{m}$, mientras la posición de $\mathrm{e}_{2}$ varía. Se observa que la duración del CAP tiene un comportamiento incremental con $\left|d_{e 1}-d_{e 2}\right|$. Es importante destacar que la simulación de cada situación experimental fue repetida 10 veces, con la intención de observar la variabilidad debido a la distribución aleatoria de las fibras sobre la superficie transversal del haz.

El incremento de la distancia entre los electrodos de registro $\mathrm{y}$ el origen de los impulsos nerviosos produce una disminución en la amplitud del CAP diferencial, mientras la duración de estos se mantiene constante (Fig. 4C). Al mismo tiempo se observa la aparición de picos secundarios debido al aporte de fibras de menor diámetro (menor velocidad de propagación). Estas simulaciones fueron realizadas considerando una conductividad longitudinal del intersticio tal que el electrodo de registro es capaz de capturar la actividad de una fibra en contacto hasta a una distancia de $10000 \mu \mathrm{m}$ hacia ambos lados del mismo (equivalente a 5 nodos de Ranvier hacia cada lado). La variación de esta característica (indirectamente relacionado con la conductividad longitudinal del intersticio) produce variaciones de amplitud y duración en los CAPs diferenciales simulados (Fig. 4D). Estos cambios son atribuidos a la cantidad de impulsos nerviosos capaz de capturar un electrodo de registro.

La Fig. 4E muestra los CAPs diferenciales simulados para impulsos nerviosos de duración incremental. Estas simulaciones, en particular, fueron tenidas en cuenta debido a que la morfología del impulso nervioso (potencial registrado en un nodo) depende de las características morfológicas del nervio. La amplitud de los $\mathrm{V}_{\mathrm{m}}$, en todos los casos se mantuvo constante (igual a uno).

El incremento de la conductividad transversal, la cual está relacionado a la cantidad de fibras activas capturadas un electrodo de registro, evoca una disminución en la amplitud relativa de los CAPs diferenciales (Fig. 4F). La duración de estos se mantiene sin cambios significativos.

La Fig. 5A muestra las simulaciones obtenidas en el nervio infraorbitario, teniendo en cuenta características morfológicas específicas. Debido a que la distancia entre nodos de Ranvier no se encuentra en la bibliografía citada, hemos considerando: $500,1000,1500$ y $2000 \mu \mathrm{m}$. La duración del impulso nervioso es constante e igual a $0.5 \mathrm{~ms}$. Los CAPs diferenciales así

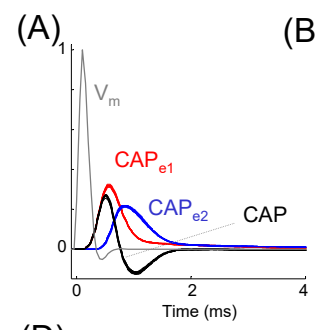

(D)
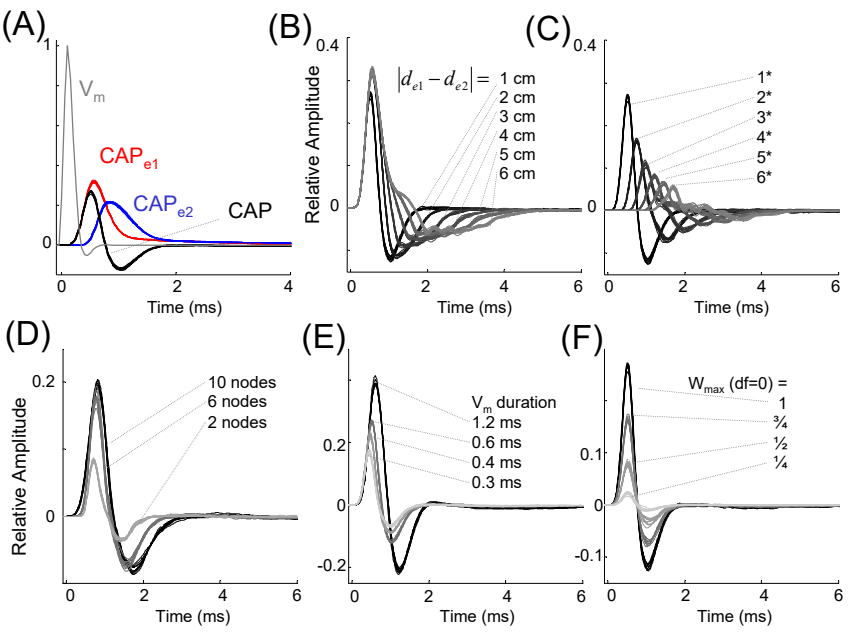

(E)

(F)

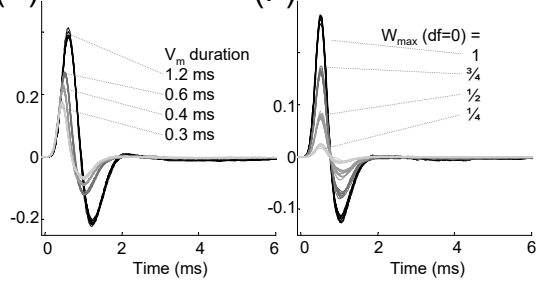

Figura 4. Simulaciones en el nervio ciático. (A) CAPs obtenidos a través de los electrodos de registro (rojo y azul) y CAP diferencial (negro). En gris se observa el impulso nervioso que viaja a lo largo de cada una de las fibras del nervio. Las amplitudes de los CAPs son relativas a la amplitud de $\mathrm{V}_{\mathrm{m}}$. Las características morfológicas del nervio simulado fueron: diámetro del haz $1500 \mu \mathrm{m}, 10$ nodos, $\mathrm{d}_{\mathrm{n}}=2000 \mu \mathrm{m}$, posiciones de $\mathrm{e}_{1} \mathrm{y} \mathrm{e}_{2}, 10000$ y $20000 \mu \mathrm{m}$, respectivamente. En todos los casos 10 simulaciones fueron realizadas. (B) Variación de la distancia inter-electrodo. La posición del electrodo e1 no varía y es $10000 \mu \mathrm{m}$. (C) CAPs diferenciales obtenidos desde los electrodos colocados a diferentes distancias. (D) CAPs diferenciales obtenidos para diferentes aperturas longitudinales de $\mathrm{Wj}$. Las aperturas longitudinales son consideradas tal que abarcan la actividad de 10, 6 y 2 nodos. (E) CAPs diferenciales para Vm de diferentes duraciones. (F) CAPs diferenciales para diferentes valores $\alpha$.

simulados muestran variaciones de amplitud y duración. Finalmente, los CAPs registrados experimentalmente pueden ser descritos por el modelo propuesto tal como se muestra en la Fig. 5B. Es importante destacar que las simulaciones realizadas requieren el conocimiento previo de la velocidad de propagación promedio, la cual, en este trabajo, fue experimentalmente determinada empleando el protocolo descrito en la sección de Materiales y Métodos.

\section{DISCUSIONES}

La caracterización electrofisiológica más ampliamente utilizada, en docencia e investigación, es frecuentemente practicada en el nervio ciático de sapo. Su implementación permite exponer conceptos fundamentales de la electrofisiología clásica [1], a la vez que facilita la validación de nuevos protocolos de registro, estudios sobre estimulación nerviosa y modelos matemáticos / computacionales que describen la génesis y dinámica del CAPs [13]. En este trabajo hemos simulado diferentes condiciones experimentales utilizadas en la caracterización electrofisiológica del nervio ciático [15]. La validación de las simulaciones obtenidas, es realizada a través de la bibliografía dedicada a procedimientos y protocolos para el registro del CAP en fibras nerviosas.

Tras establecer una morfología predeterminada para el impulso nervioso, $\mathrm{V}_{\mathrm{m}}$, el cual se propaga a lo largo de cada una de las fibras del haz, con perfiles de velocidad específicos 
(directamente relacionados al diámetro), fue posible determinar los CAPs registrados a través de electrodos extracelulares en configuraciones monopolar y diferencial. Las simulaciones realizadas revelan una forma de onda del CAP, la cual se ajusta adecuadamente a datos experimentales [16]. Sin embargo, es importante destacar que el modelo propuesto supone un contacto puntual con impedancia de interfase nula, lo cual simplifica considerablemente su formulación. Sin embargo, un mejor ajuste podría realizarse a través de una aproximación no-lineal, la cual modele un área de contacto, por ejemplo, haciendo uso de la teoría de interfase electrodo-electrolito [17].

La modelización de situaciones, en las cuales se varían las distancias entre los electrodos de registro y estimulación, permitiría al experimentador predecir los posicionamientos adecuados con el fin de observar la contribución de fibras más lentas [18]. En las simulaciones de la Fig. 4B y 4C se observa esta particularidad. El alejamiento del electrodo $\mathrm{e}_{2}$ revela la contribución de fibras de menor diámetro en el CAP registrado [15]. Por otro lado se observa una disminución en la amplitud del CAP, con el aumento de la distancia entre los electrodos de registro y estimulación. Teóricamente, este comportamiento se debe a que los impulsos nerviosos son conducidos por las fibras nerviosas a diferentes velocidades. Esto genera, en los electrodos de registro, agrupamientos de SFAP cada vez más diferenciales [18]. Sin embargo, Pehlivan et al. [19], demostró que los cambios de amplitud, también pueden ser atribuidos a la variabilidad existente de la distribución anatómica de fibras a lo largo del haz nervioso. En este sentido, el modelo propuesto podría explicar parcialmente esta particularidad, a través del análisis de las variaciones de amplitud del CAP, tanto en el modelo como aquellos observados experimentalmente. Luego, las diferencias entre ambas observaciones podrían revelar las contribuciones debido a las irregularidades anatómicas del nervio.

Wijesinghe et al. [13] demostró, teórica y experimentalmente, que la forma de onda del CAP presenta cambios de amplitud cuando varía la conductancia del intersticio. El modelo propuesto ha reproducido adecuadamente estos cambios (Fig. 4D y 4F), a través de variaciones cualitativas en las conductividades longitudinal y transversal. Es importante destacar que las simulaciones realizadas se basan en propiedades ideales de conducción: medio conductor homogéneo y resistivo. Sin embargo, es bien conocido que los medios de conducción biológicos presentar un alto grado de anisotropía, involucrando así, formulaciones no lineales en su modelización [20]. Otras características anatómicas, las cuales producen cambios morfológicos en los potenciales del haz, son las dimensiones intrínsecas de las fibras que lo componen. Así por ejemplo, la velocidad de conducción del impulso nervioso está directamente relacionada con el diámetro de la fibra [1]. Contento et al. [9], demostró además, que variaciones morfológicas del SFAP afectan significativamente al CAP. Para comprobar la versatilidad de nuestra implementación, hemos simulado variaciones intrínsecas del impulso nervioso $\left(\mathrm{V}_{\mathrm{m}}\right)$, bajo la hipótesis de que estos potenciales cambian la forma de onda del SFAP, y hemos obtenido los CAPs resultantes. Los resultados han mostrado variaciones de amplitud y duración en los CAPs, conforme a diferentes duraciones de $\mathrm{V}_{\mathrm{m}}$. Por otro lado, el modelo permitiría, además, cambiar la forma de onda de $V_{m}$ (no solo parámetros temporales) y determinar cambios cualitativos en los CAPs. Esta particularidad, le agrega al modelo una mayor flexibilidad, junto con la capacidad de adecuarse a diferentes hipótesis experimentales.

La validación experimental del modelo, fue realizada a través de un protocolo estándar para la generación del CAP en el nervio infraorbitario de rata. Los datos anatómicos del nervio IO han sido bien documentados por Jacquin et al. [14], no así su caracterización electrofisiológica. Las simulaciones realizadas ajustan adecuadamente los resultados experimentales (Fig. 5B), sin embargo algunas consideraciones deben tenerse en cuenta. En primer lugar, el modelo propuesto requiere el conocimiento previo de ciertas características anatómicas del nervio, tales como diámetros de las fibras, distancia entre nodos y velocidad de conducción. En particular, la distancia entre fibras no fue documentada por Jacquin et al. [14], por lo que se tuvieron que implementar diferentes valores. A través de un análisis cualitativo se determinó que el mejor ajuste resulta para una distancia entre nodos de $500 \mu \mathrm{m}$. Nuevamente, el modelo puede predecir con gran precisión las características temporales del CAP, mientras que las características de amplitud solo pueden ser cuantificadas respecto a la amplitud del impulso nervioso (amplitudes relativas).
(A)

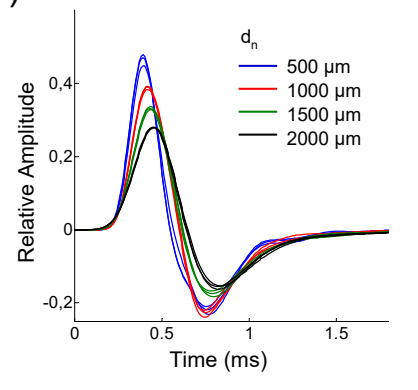

(B)

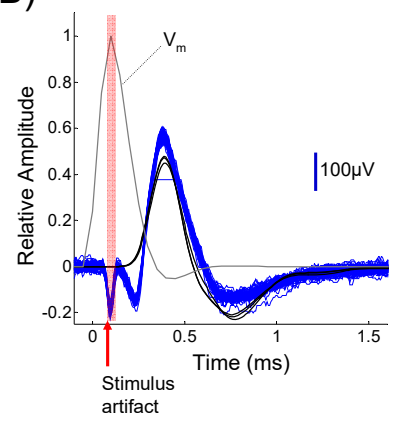

Figura 5. Simulaciones en el nervio infraorbitario y registros experimentales. (A) CAPs diferenciales obtenidos para fibras con diferentes distancias entre nodos. Tres simulaciones fueron realizadas para cada caso. Las amplitudes de los CAPs son relativas al impulso nervioso (Vm). Las especificaciones comunes de estas simulaciones fueron: diámetro del haz $1265 \mu \mathrm{m}, 10$ nodos, $\mathrm{dn}=$ variable, posiciones de e1 y e2, 12000 y $16000 \mu \mathrm{m}$, respectivamente. (B) 40 registros CAPs diferenciales obtenidos experimentalmente (azul) y 3 CAPs simulados (líneas de trazo negro). El impulso nervioso coincide

\section{CONCLUSIONES}

El modelo propuesto reúne, de manera cualitativa y cuantitativa, las características más relevantes de la conducción nerviosa en fibras mielínicas, permitiendo así parametrizar, de manera simplificada e intuitiva, condiciones experimentales reales. Permite la entrada de parámetros simples, los cuales pueden representar condiciones intrínsecas y dinámicas de la conducción nerviosa. La validación experimental ha demostrado un adecuado ajuste del modelo. Finalmente, la evaluación global del modelo, revela importantes características de simplicidad, versatilidad y robustez, las cuales pueden ser aprovechadas tanto en áreas 
vinculadas a la educación, como a la experimentación científica.

\section{AGRADECIMIENTOS}

Este trabajo ha sido financiado por la Agencia Nacional de Promoción Científica y Tecnológica (ANPCYT), Consejo Nacional de Investigaciones Científicas y Técnicas (CONICET), y el Consejo de Investigaciones de la Universidad Nacional de Tucumán (CIUNT), como así también por fondos institucionales del Instituto Superior de Investigaciones Biológicas (INSIBIO).

\section{REFERENCIAS}

[1] T. C. Ruch, Physiology and biophysics, 20th ed. Philadelphia: W.B. Saunders Company, 1973.

[2] F. Buchthal and A. Rosenfalck, "Evoked action potentials and conduction velocity in human sensory nerves," Brain Research, vol. 3 , no. 1, pp. v-122, Nov. 1966.

[3] R. Schoonhoven, D. F. Stegeman, and A. van Oosterom, "The Forward Problem in Electroneurography II: Comparson of Models," IEEE Transactions on Biomedical Engineering, vol. BME-33, no. 3 , pp. 335-341, Mar. 1986.

[4] A. T. Barker, B. H. Brown, and I. L. Freeston, "Modeling of an Active Nerve Fiber in a Finite Volume Conductor and Its Application to the Calculation of Surface Action Potentials," IEEE Transactions on Biomedical Engineering, vol. BME-26, no. 1, pp. 53-56, Enero 1979.

[5] R. Schoonhoven, D. F. Stegeman, and J. P. C. DE Weerd, "The Forward Problem in Electroneurography I: A Generalized Volume Conductor Model," IEEE Transactions on Biomedical Engineering, vol. BME-33, no. 3, pp. 327-334, Mar. 1986.

[6] L. N. S. Andreasen and J. J. Struijk, "Signal strength versus cuff length in nerve cuff electrode recordings," IEEE Transactions on Biomedical Engineering, vol. 49, no. 9, pp. 1045-1050, Sep. 2002.

[7] N. M. Bogatov, L. R. Grigoryan, E. G. Ponetaeva, and A. S. Sinisyn, "Calculation of action potential propagation in nerve fiber," Prog. Biophys. Mol. Biol., vol. 114, no. 3, pp. 170-174, May 2014.

[8] D. J. Caddy, H. R. Kranz, and R. A. Westerman, "Electrophysiological determination of peripheral nerve conduction velocity distribution," in New Approaches to Nerve and Muscle Disorders. Basic and Applied Contributions, vol. 5, A. D. Kidman, J. K. Tomkins, and R. A. Westerman, Eds. Amsterdam, The Netherlands: Elsevier, 1981, p. 418.

[9] G. Contento, V. Barbina, M. R. Malisan, R. Padovani, R. Budai, and I. Pittaro, "Dependence of the linear model for the nerve compound action potential on the single fibre action potential waveform," Clin Phys Physiol Meas, vol. 4, no. 4, pp. 417-433, Nov. 1983.

[10] L. Goldman and J. S. Albus, "Computation of Impulse Conduction in Myelinated Fibers; Theoretical Basis of the Velocity-Diameter Relation," Biophys J, vol. 8, no. 5, pp. 596-607, May 1968.

[11] S. Reutskiy, E. Rossoni, and B. Tirozzi, "Conduction in bundles of demyelinated nerve fibers: computer simulation," Biol Cybern, vol. 89, no. 6, pp. 439-448, Dec. 2003.

[12] T. Wang and Z. Fan, "A modified bidomain model for extracellular potential of the active bundle of compound nerve fibres," in 1992 14th Annual International Conference of the IEEE Engineering in Medicine and Biology Society, 1992, vol. 2, pp. 827-828.

[13] R. S. Wijesinghe, F. L. Gielen, and J. P. Wikswo, "A model for compound action potentials and currents in a nerve bundle. I: The forward calculation," Ann Biomed Eng, vol. 19, no. 1, pp. 43-72, 1991.
[14] M. F. Jacquin, A. Hess, G. Yang, P. Adamo, M. F. Math, A. Brown, and R. W. Rhoades, "Organization of the infraorbital nerve in rat: a quantitative electron microscopic study," Brain Res., vol. 290, no. 1, pp. 131-135, Jan. 1984.

[15] J. Erlanger and H. S. Gasser, Electrical signs of nervous activity, 1st ed. Pennsylvania, USA: University of Pennsylvania Press, 1937.

[16] R. M. Silverman and P. M. Brunett, The Compound Action Potential: An Introduction of Principles of Neurophysiology. Phipps \& Bird, Incorporated, 1978.

[17] G. Ruiz and C. J. Felice, "Non-linear response of an electrodeelectrolyte interface impedance with the frequency," Chaos, Solitons \& Fractals, vol. 31, no. 2, pp. 327-335, Enero 2007.

[18] A. J. McComas, Galvani's Spark: The Story of the Nerve Impulse. New York, USA: Oxford University Press, 2011.

[19] F. Pehlivan, N. Dalkilic, and E. Kiziltan, "Does the conduction velocity distribution change along the nerve?," Medical Engineering \& Physics, vol. 26, no. 5, pp. 395-401, Jun. 2004.

[20] K. W. Altman and R. Plonsey, "Analysis of the longitudinal and radial resistivity measurements of the nerve trunk," Ann Biomed Eng, vol. 17, no. 4, pp. 313-324, Jul. 1989.

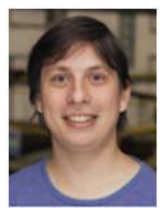

A. Gabriel Pizá Es ingeniero Biomédico de la Universidad Nacional de Tucumán (2012), está realizado sus estudios de doctorado desde el año 2013 en el área de las neurociencias. Se especializa en análisis computacional de señales electrofisiológicas.

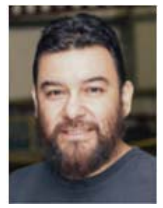

Fernando D. Farfán ingeniero electrónico y Dr. en Ciencias Biológicas de la Universidad Nacional de Tucumán. Investigador del Instituto Superior de Investigaciones Biológicas en el área de la neurociencia computacional.

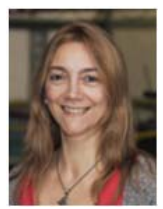

Ana L. Albarracín Bióloga y Dra. en Ciencias Biológicas de la Universidad Nacional de Tucumán. Investigador del Instituto Superior de Investigaciones Biológicas en el área de electrofisiología.

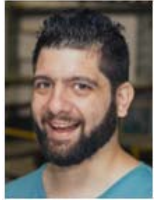

Facundo A. Lucianna Es ingeniero Biomédico de la Universidad Nacional de Tucumán (2013), está realizado sus estudios de doctorado desde el año 2013 en el área de las neurociencias. Se especializa en modelaje matemático y análisis numérico.

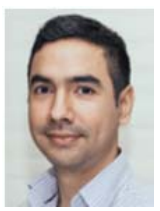

Jorge H. Soletta Es ingeniero Biomédico de la Universidad Nacional de Tucumán (2011), está realizado sus estudios de doctorado desde el año 2012 en el área de las neurociencias. Se especializa en el estudio de la visión.

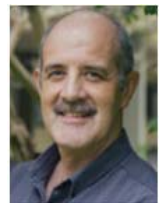

Carmelo J. Felice ingeniero en electrónica y Doctorado en la Universidad Nacional de Tucumán. Investigador del Instituto Superior de Investigaciones Biológicas en el área de bioimpedancimetría. 